\title{
Comparison of Hertzian and JKR theories with a finite element model in boundary lubrication conditions between a compression ring and a cylinder
}

\author{
Kyriakos Grigoriadis ${ }^{1 *}$, Anastasios Zavos ${ }^{1}$, and Pantelis G. Nikolakopoulos ${ }^{l *}$ \\ ${ }^{1}$ Machine Design Laboratory, Dept. of Mechanical Engineering and Aeronautics, \\ University of Patras, Patras 26504, Greece
}

\begin{abstract}
This study focuses on the creation of an isothermal elastic model to highlight, through stresses, the occurrence of plastic deformation in certain crank angles under extreme dry conditions inside an internal combustion engine. The stresses that are exported from this analysis are pointing out not only the necessity for an elastoplastic model to be created, but also the importance of predicting the correct friction coefficient, as pointed out by both the contact surface stress and those in depth of the two bodies in contact. A comparison between two coefficients of frictions and one frictionless case is conducted. The comparison between the finite element model and the adhesion mathematical model of Johnson, Kendall and Roberts (JKR), seals the importance of the interaction forces, acting on the common solid surface, in the pursuit of defining a propriate contact patch. Furthermore, a three-dimensional model is proposed for further investigation, highlighting the importance of modelling surface's micro asperities for a solid stress analysis.
\end{abstract}

\section{Introduction}

Recent comprehensive study by Holmberg et al. [1] showed that energy losses are the highest in engines and transmissions due to contact. Among these, the ring-pack assembly is the primary source of friction [2]. This contact is also one of the most complex to analyse because of the transient nature of this conjunction and the variation of the regimes of lubrication. The basic theory of contact between elastic bodies has been of great importance for many years. Initially, variety of models like Johnson [3] and Hills et al. [4] approach consistently the mechanical problem of contact while, Gladwell [5] and Galin [6] along with Kikuchi et. al [7] and Khludnev and Sokolowski [8], reported the same problem from both a mathematic and a finite element perspective, respectively. Additionally, Bhushan conducted research for both a single (Hertzian) [9] and multiple asperity [10] approach laying the foundations of the so-called contact mechanics of rough surfaces field. Schwarz [11] was also focused on calculating the elastic deformation of a sphere on a flat surface

${ }^{1}$ Corresponding author: Pantelis G. Nikolakopoulos, e-mail: pnikolakop@upatras.gr 
with JKR and DMT models while Adams and Nosonovsky [12] categorized the theoretical contact models according to adhesion, friction and single or multi asperity. Shi and Zhao [13] compared the adhesion JKR and DMT models with the adhesion less Hertzian model mentioning the influence of dimensionless load parameter. A complete research in the field of surface energy, which is the factor that distinguishes the JKR from the Hertzian model, was conducted by Leite et al. [14] provided an extended list of measured materials in terms of intermolecular interactions and surface forces along with a complete theoretical approach.

The pioneering work of Chong and Fong [15] proposed a deterministic friction force predictive model which included a fluid film shear component along with a possible asperity contact for a ring-liner conjunction on top dead center, inside an internal combustion engine. The author addressed the boundary friction using a multi-scale model, aims to provide the mechanisms underlying friction at asperity level between rough surfaces.

Our approach is coming to an end for the creation of an isothermal elastic model to highlight, stresses distribution in certain crank angles under extreme dry conditions. The stresses that are exported from this analysis are pointing out not only the necessity for an elastoplastic model to be created, but also the importance of predicting the correct friction coefficient, as pointed out by both the contact surface and in depth of the two bodies in contact stresses. A comparison between two coefficients of frictions and one frictionless case is conducted. Furthermore, the comparison between the finite element model and the mathematical model of Johnson, Kendall \& Roberts, seals the importance of the interaction forces, acting on the common solid surface, in the pursuit of defining a propriate contact patch. Furthermore, a three-dimensional model is proposed for further investigation, highlighting the importance of modelling surface's micro asperities for a solid stress analysis.

\section{Background Theory}

\subsection{Surface energy-Work of adhesion}

The difference between the two compared mathematical models (Hertzian and JKR) is on the interaction forces that are considered to act in the contact surface of the compression ring and the cylinder liner. In this case, in which the contact between two surfaces is elastic, results to no energy dissipation during the interaction and so both the adhesive and the surface forces are equal in magnitude [11]. Schwarz mentioned that the area between the force curve and the base line right from the equilibrium distance $z_{0}$ is considered as the work of adhesion or Dupré's energy [11]:

$$
\gamma=\int_{z_{0}}^{\infty} \sigma(z) d z
$$

where $\sigma(z)$ is the force per unit area. Lennard-Jones potential typically represents the adhesive stress $(z)[11]$,

$$
\sigma(z)=-\frac{8 \gamma}{3 z_{0}}\left[\left(\frac{z}{z_{0}}\right)^{-3}-\left(\frac{z}{z_{0}}\right)^{-9}\right]
$$

where $z$ is the separation between atomic planes.

Another method consists in calculating the work of adhesion and then relating it to the Hamaker constant through [12]. Separation will occur only when contact area $a^{J K R}$ 
becomes equal to $0.63 a_{o}^{J K R}$ where $a_{o}^{J K R}$ is the contact area in absence of external load. If two phases $(i, j)$ in contact are pulled apart inside a third phase $k$ then the work of adhesion according to [12] is given by the equation (4),

$$
\gamma=\gamma_{i k}+\gamma_{j k}-\gamma_{i j}
$$

Another method consists in calculating the work of adhesion and then relating it to the Hamaker constant through [14]. Separation will occur only when contact area $a^{J K R}$ becomes equal to $0.63 a_{o}^{J K R}$ where $a_{o}^{J K R}$ is the contact area in absence of external load. If two phases $(i, j)$ in contact are pulled apart inside a third phase $k$ then the work of adhesion according to [14] is given by the expression (4):

$$
\gamma=\gamma_{i k}+\gamma_{j k}-\gamma_{i j}
$$

As an attempt to build an adhesive contact model, the work of adhesion parameter used to model JKR was obtained from Chong et al. [15].

\subsection{Adhesionless single asperity contact-Hertz model}

The two bodies, the compression ring and the cylinder liner can be treated as twodimensional cylindrical contact problem, in top and bottom dead center of a four-stroke cycle, always absence of lubricant. Stachowiak and Batchelor [16] formulated the basic contact parameters between two parallel cylinders. It is assumed that the dimensions of the contact area are small compared to the dimensions of each body and to the radii of curvature of the surfaces and that the strains are sufficiently small for linear elasticity to be valid and. Neglecting any attractive forces $(\gamma=0)$ and considering only the external load acting in the back of the ring, the contact radius, maximum deflection and maximum contact pressure, which occurs at $r=0$ (center of contact), are predicted from the Hertzian model as follows [17]:

$$
\begin{gathered}
a=\left(\frac{4 P R}{\pi L E^{*}}\right)^{1 / 2} \\
\delta=0,319\left(\frac{P}{E^{*} L}\right) \times\left[\frac{2}{3}+\ln \left(\frac{4 R_{1} R_{2}}{a^{2}}\right)\right] \\
p_{0}^{H}=\frac{P}{\pi a L} \\
\tau_{\text {max }}=0,304 p_{0}^{H} \\
\text { at depth of, } z=0,786 a
\end{gathered}
$$

$$
\text { where } \frac{1}{E^{*}}=\frac{1-v_{1}^{2}}{E_{1}}+\frac{1-v_{2}^{2}}{E_{2}} \quad(10) \text { and } \quad \frac{1}{R}=\frac{1}{R_{1}}+\frac{1}{R_{2}}
$$

with $E_{1}, E_{2}, v_{1}, v_{2}$ being the Young's modulus and Poisson's ratio for the compression ring and the cylinder liner respectively, $\delta$ is indentation depth and $\alpha$ is the radius of contact area. $E^{*}$ is the reduced Young's modulus and $R$ the effective radius of the two cylinder bodies, with $R_{2}=\infty$ for the infinite cylinder radius (the cylinder is considered as plane).

The pressure distribution over the contact area, considering equally distributed external load calculated by the equation (12): 


$$
p^{H}(r)=p_{0}^{H}\left(1-\frac{r^{2}}{a^{2}}\right)^{1 / 2}
$$

where $r^{2}=x^{2}+y^{2}$, although considering two-dimensional model, $r=x$.

\subsection{Adhesion single asperity contact-JKR model}

Extending the Hertzian theory and setting $\gamma \neq 0$ the contact equations are modified including the effect of the work of adhesion. The effective external load applied according to the work of Johnson et al. [11] is given on equation (13):

$$
p^{J K R}=P+3 \pi R \gamma \pm \sqrt{6 \pi R \gamma P+(3 \pi R \gamma)^{2}}
$$

which is larger than the external force used in Hertzian approximation. The minus in the solution denotes unstable conditions. Contact radius, maximum deflection and maximum contact pressure, which are dependent on the external applied load are given by equations (14), (15) and (16):

$$
\begin{aligned}
& \alpha^{J K R}=\left(\frac{R P^{J K R}}{K}\right)^{1 / 3} \\
& \delta^{J K R}=\frac{a^{2}}{R}-\sqrt{\frac{8 \pi \gamma a}{3 K}} \\
& p_{0}^{J K R}=\left(\frac{3 \gamma K}{2 \pi a}\right)^{1 / 2} \\
& \text { where } K=\frac{4 E^{*}}{3} .
\end{aligned}
$$

Compared with the Hertzian approach, the pressure distribution over the cyclic contact area, considering equally distributed external load is:

$$
p^{J K R}(r)=p^{H}(r)-p_{0}^{J K R}\left(1-\left(\frac{r}{a}\right)^{2}\right)^{-1 / 2}
$$

\section{Finite Element Modelling}

\subsection{Two-Dimensional Elastic Model}

The contact performance of the top compression ring-liner conjunction for a high performance four-stroke engine at dead centers is studied through numerical analysis. The material of the ring is Steel SAE 925 and its mechanical properties received from the ref. [18]. Both the cylinder and the compression ring are deformable solids. Table 1 presents the basic dimensions. The forces acting on the back of the ring in radial plane, are the ring's elastic tension force and the gas force, both obtained from the work of Shahmohamadi et al. [18].

Table 1. Ring-liner tribo-pair dimensions.

\begin{tabular}{|c|c|c|}
\hline Parameter & Compression Ring & Cylinder Liner \\
\hline Axial face width $(\mathrm{m})$ & $1.15 \times 10^{-3}$ & $1.15 \times 10^{-3}$ \\
\hline Radial face width $(\mathrm{m})$ & $0.085 \times 10^{-3}$ & $0.085 \times 10^{-3}$ \\
\hline
\end{tabular}




\begin{tabular}{|c|c|c|}
\hline Crown height $(\mathrm{m})$ & $10 \times 10^{-6}$ & - \\
\hline
\end{tabular}

A total of 33810 quadrilateral elements are used and a Penalty method algorithm solves the contact problem in ANSYS Multiphysics package. The analyses are conducted for nonlinear geometrical behavior of the finite element whose stiffness matrix is given by the nonlinear equation (19):

$$
K(u)=K_{0}\left(1-\frac{u}{U}\right)=\text { stifness matrix }
$$

where $K_{0}$ is the initial contact stiffness $(\mathrm{N} / \mathrm{m}), U$ is the sliding velocity $(\mathrm{m} / \mathrm{s})$ and $u$ is the nodal momentarily velocity $(\mathrm{m} / \mathrm{s})$. The initial stiffness suggested to take values in an order of magnitude larger than the elastic modulus of the underlying geometry. All and all this value must be a balanced value between the accuracy of the results and the run time.A comparison is conducted for coefficients of friction, $\mu=0$ and $\mu=1$ for examination of the equivalent normal; stresses along the depth of the compression ring. Solving iteratively, using the Newton Raphson Method. the equations of the stresses equilibrium, the vertical stress distribution along piston ring's depth for friction, frictionless and analytical solution is plotted in Fig.1.

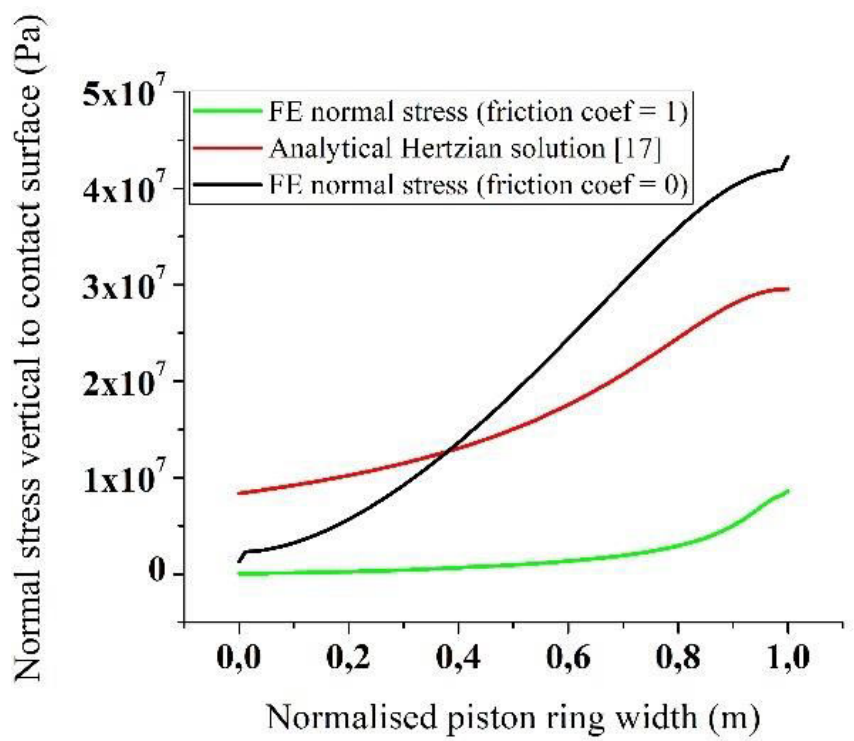

Fig. 1. Normal stress in vertical direction along compression ring's depth.

Comparing the case of analytical (line contact in two cylinders with parallel axis) and frictional solution, a lack of convergence is spotted. Both analytical and frictionless cases do match with the friction one in the boundary lubrication of the contact surface and in the back surface of the ring, respectively. As we approach the highest contact point between the two contact surfaces (Y-cord $=10-4)$, a significant deflection in normal mechanical stress between the two cases is observed. In the frictionless case (sliding surfaces), thermal load is neglected therefore, stress is fully translated into mechanical (shear), while in the case of $\mu=1$ (sticking) a great amount of energy is converted into thermal stresses (which are not presented in the current research work) justifying the lower mechanical stress value, and denotes the pure contact in dead centers of an internal combustion engine. 


\subsection{Boundary conditions}

Fig. 2 shows the simulation model for ring-liner contact. A first step for every finite element model is to validate an existed theoretical model. Therefore, an analysis should be conducted in absence of any axial forces and considering only the radial ones acting on the ring. As a result, a pressure load is acting on the back of the ring while cylinder's back is fixed. The number of elements used was 38.410 and the number of nodes was 38.577. The convergence was achieved using the CONTACT172 and TARGE169 type of elements.

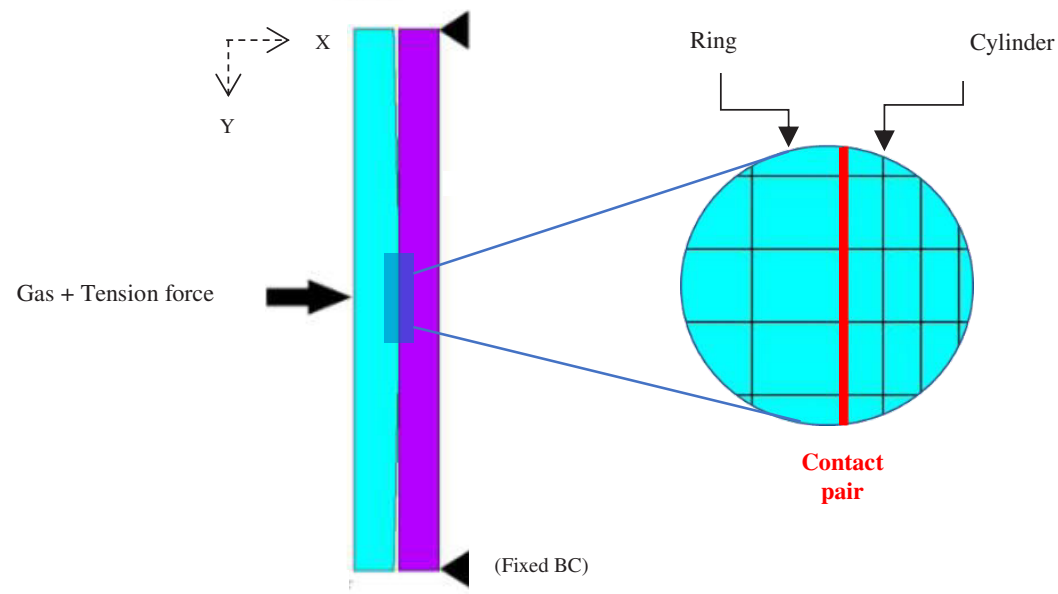

Fig. 2. Input boundary conditions and meshing details.

\section{Results and Discussion}

\subsection{Comparing Hertzian and JKR theoretical model with the finite element model}

After examining the dead center angles of every stroke $\left(-180^{\circ}, 0^{\circ}, 180^{\circ}, 360^{\circ}\right.$ and $\left.540^{\circ}\right)$, a comparison was conducted for $0^{\circ}$ which turned out to be the most critical among them [18]. In Fig. 3 is presented the contact pressure distribution over the normalized contact area for the Hertzian, JKR and FE models. The deviation of models is approximately $10 \%$, at one hand, owing to the absence of surface energy in case of the Hertzian solution and on the other hand owing to dependence of the FEA model from the number of its elements. Furthermore, the equations formulating both Hertzian and JKR theory refer to cylindrical geometry, which slightly differs from our model's case. However, the maximum value of the contact pressure has a good agreement for three models. In order to get the most accurate results, grid sensitivity tests were performed. 


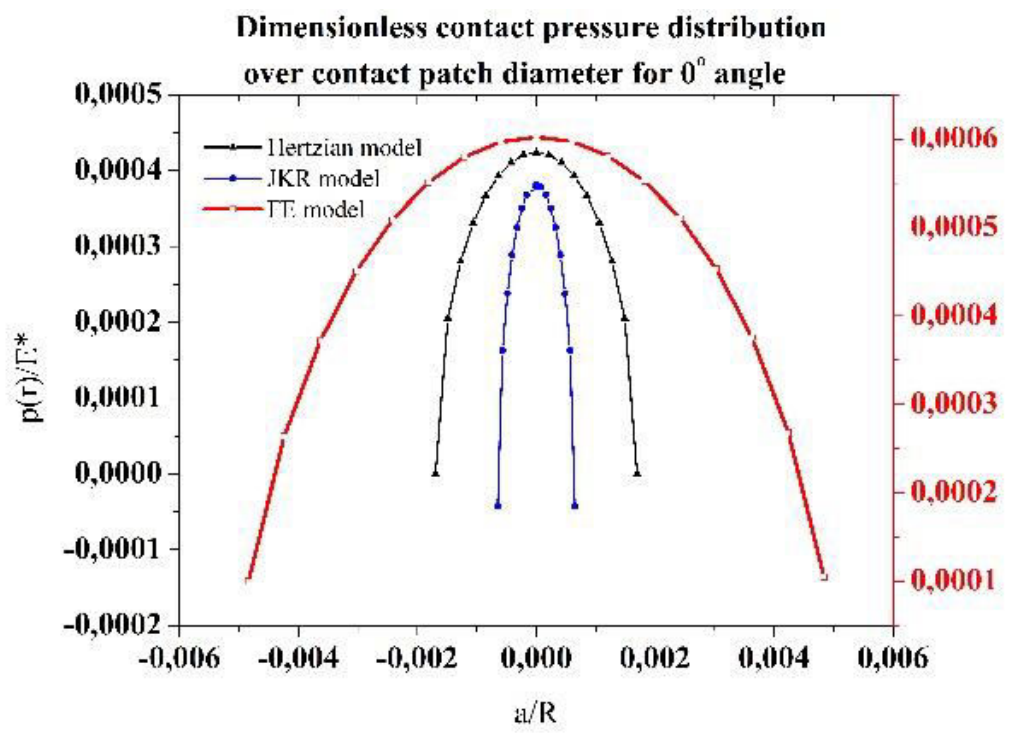

Fig.3. Comparison between the Hertzian theory, the JKR theory and the FEA.

\subsection{The effect of Gaussian distributed ring surface roughness on contact stresses}

A numerical three-dimensional model with Gaussian distributed surface roughness was created, simulating a cylinder liner and piston ring contact pair in a small area of their contact patch on microscale (Fig. 4). The model was created using a 3D sample, $9 \times 9 \times$ $3 \mathrm{~mm}$ with roughness distribution according to Gauss statistical distribution.

Fig. 5 shows that as the asperities density becomes higher the maximum equivalent stress becomes also higher. Furthermore, an obvious reduction in maximum pressure is observed disproportionately to the asperity peaks raise, in which more surface (peaks) share an equal amount of load. Among them, was pointed out the necessity of a golden section to be found between those topographical parameters compared. The Gaussian roughness distribution (or the asperities density) was developed using a) one, b) two and c) five roughness peaks per $\mathrm{mm}$. The height of the roughness was variated as, $0.02-10^{-4}, 0.2-10^{-4}$ and $10^{-4} \mathrm{~mm}$. The material was Steel SAE 9254, and the problem run for $6000 \mathrm{rpm}$, and surface to surface contact model using TARGE170 and CONTA174 type of elements.

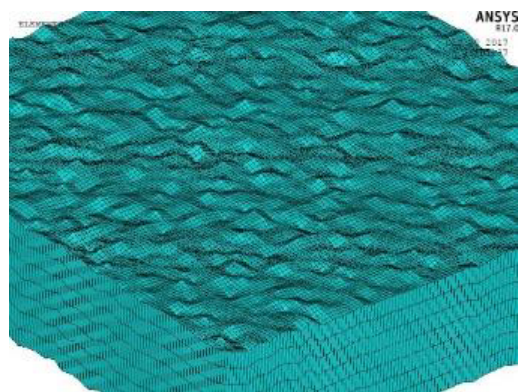

Fig. 4. Indicative surface roughness distribution on compression ring surface. 
a)

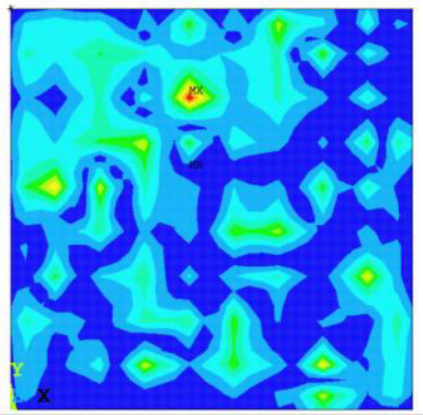

b)

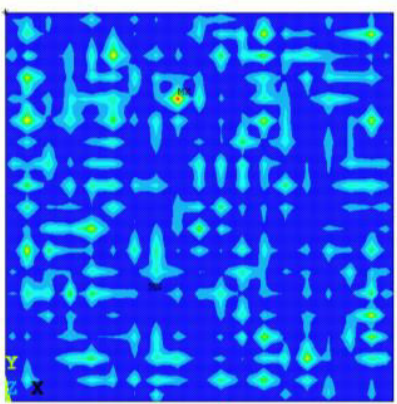

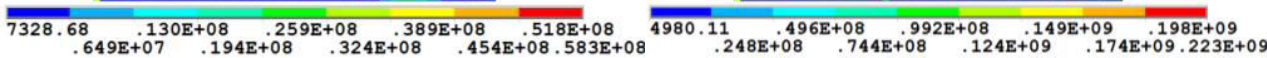

c)

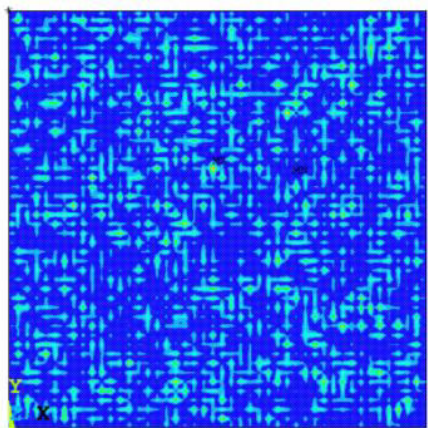

$\begin{array}{rrrrr}785.634 & .115 \mathrm{E}+09 & .230 \mathrm{E}+09 & .345 \mathrm{E}+09 & .460 \mathrm{E}+09 \\ .575 \mathrm{E}+08 & .173 \mathrm{E}+09 & .288 \mathrm{E}+09 & .403 \mathrm{E}+09.518 \mathrm{E}+09\end{array}$

Fig. 5. 3D contact modelling total stresses on compression ring surface for three different asperity dense contact surfaces: a) one peak per $\mathrm{mm}, \mathrm{b}$ ) two peaks per $\mathrm{mm}$, c) five peaks per $\mathrm{mm}$ for an asperity mean height of $0.2 \times 10^{-4} \mathrm{~mm}$.

\section{Conclusion-Future Research}

The main conclusions can be summarized here:

- The maximum value of the contact pressure has a good agreement for three models which declares the accuracy of both an adhessionless (Hertz) and an adhesion (JKR) model (Fig. 3).

- Regarding the three-dimensional model, a remarkable reduction in maximum pressure is observed due to the asperity peaks raise, in which more surface (peaks) share an equal amount of load. Additionally, considering both the error of nonlinear calculations inside the contact area, which increases proportionally to the number of elements, and also the unpredictable nature of asperity distribution, a necessity for finding the "golden ratio" between density and mean peak height parameters, is observed (Fig.4).

The ability to predict the extend adhesion forces between two contact surfaces, on a multiasperity model, is a step further of this work. The immediate affection on the existing single asperity models like the ones of DMT [19], JKR [20], and Maugis-Dugdale [21] should also be investigated.

\section{References}


1. K. Holmberg, A. Erdemir Friction, 5, 263-284,2017.

2. A. Zavos, P.G. Nikolakopoulos, Proceedings of the Institution of Mechanical Engineers, Part D: Journal of Automobile Engineering, 0954407017734770.

3. KL. Johnson, Contact mechanics, Cambridge: Cambridge University Press, 1985

4. DA. Hills, D. Nowell, A. Sackfield, Mechanics of elastic contact, Oxford: ButterworthHeinemann Ltd, 1993.

5. GML. Gladwell, Maryland, USA: Sijtoff and Noordhoff, 1980.

6. LA. Galin, Gostehizdat, Moscow (in Russian.), 1953. English translation, Sneddon IM. editor. Raleigh: North Carolina State College, 1961.

7. N. Kikuchi, JT. Oden, Philadelphia: SIAM, 1985.Galin LA. Contact problems in the theory of elasticity, Gostehizdat, Moscow (in Russian.), 1953. English translation, Sneddon IM. editor. Raleigh: North Carolina State College, 1961.

8. AM. Khludnev, J. Sokolowski, Basel: Birkhauser Verlag, 1997.Galin LA. Contact problems in the theory of elasticity, Gostehizdat, Moscow (in Russian.), 1953. English translation, Sneddon IM. editor. Raleigh: North Carolina State College, 1961.

9. B. Bhushan. Contact mechanics of rough surfaces in Tribology: single asperity contact. Appl Mech Rev, 49,275-98, 1996.

10. B. Bhushan, Tribol Let; 4, 1-35, 1998.

11. U.D. Schwarz, Journal of Colloid and Interface Science, 261, 99-106, 2003.

12. G.G Adams, M. Nosonovsky, Tribology International, 33, 431-442, 2000.

13. X. Shi, Y.P. Zhao, J. Adhesion Sci. Technol. 18, 55-68, 2003.

14. F.L. Leite, C.C. Bueno, A.L. Da Róz, E.C. Ziemath, O.N. Oliveira Jr, Int. J. Mol. Sci., 13, 12773-12856, 2012.

15. W.W.F. Chong. PhD Thesis, Cranfield University, 2012.

16. G. W. Stachowiak, A. W. Batchelor, Engineering Tribology. Butterworth Heinemann,1993.

17. H. Hertz, Über die berührung fester elastischer Körper (On the contact of rigid elastic solids). In: Miscellaneous Papers. Jones and Schott, Editors, J. reine und angewandte Mathematik 92, Macmillan, London, p. 156, 1896

18. H. Shahmohamadi, R. Rahmani, H. Rahnejat, C.P. Garner and P.D King, Tribology Letters, 530,2013 .

19. B.V. Derjaguin, V.M. Muller, Y.P. Toporov. J Colloid Interface Sci, 53, 14-26, 1975.

20. K. L. Johnson, K. Kendall, and A. D. Roberts. Proc. Roy. Soc. London A, 324:301-313, 1971.

21. D. Maugis, J. Colloid Interface Sci. 150 (1992) 243-269. 